$$
\text { "giering" — 2008/4/9 — 9:45 — page 337 — \#1 }
$$

\title{
Über ähnliche Aufsatzdreiecke einer Strecke
}

\author{
OSWALd GIERING
}

Abstract. In this article we investigate (with methods of school geometry) a figure $(P Q, A B C)$ consisting of three given similar triangles $P Q A, P Q B, P Q C$ with side $P Q$ in common (Figure 1). We combine other triangles with this figure such as triangle $A B C$ which is proved to be similar to the given triangles. The incircles of three additional triangles adjacent to triangle $A B C$ will be determined.

Key words and phrases: similar triangles, incircles of triangles.

ZDM Subject Classification: G40.

Als Beitrag zu der seit Jahrhunderten lebendigen Elementargeometrie betrachten wir die - insgesamt drei - über einer festen Strecke $P Q$ nach derselben Seite errichteten, gleichsinnig ähnlichen Aufsatzdreiecke $P Q A, P Q B, P Q C$ (Abb. 1) und untersuchen mit elementaren Methoden der Schulgeometrie die so definierte Aufsatzfigur ( $P Q, A B C)$. Es handelt sich dabei um eine einfache Ergänzung zu der an geometrischen Aussagen reichhaltigen Theorie der Aufsatzdreiecke, die im sogenannten Satz von Napoleon ihren Anfang nahm ${ }^{1}$.

Wir beschreiben zunächst in Abschnitt 1. die Aufsatzfigur $(P Q, A B C)$. In 2. beweisen wir einen Streckensatz (Satz 1), der die von $P Q$ verschiedenen Seiten der Aufsatzdreiecke verknüpft. Sodann betrachten wir in 3. das Kerndreieck

${ }^{1}$ Beiträge zu diesem Themenkreis finden sich in [1], S. 1216ff., [2], S. 65ff., [3], S. 324f., [4], [6], [7], S. 100f.

Copyright (c) 2007 by University of Debrecen 


$$
\text { "giering" — 2008/4/9 — 9:45 — page 338 — \#2 }
$$

$A B C$ der Aufsatzfigur. Eine kinematische Überlegung liefert in Satz 2 die Drehstreckungsgleichheit des Kerndreiecks zu den Aufsatzdreiecken. In Satz 3 werden weitere Paare drehstreckungsgleicher, in der Aufsatzfigur enthaltener, Dreiecke beschrieben. Über den Seiten des Kerndreiecks werden in 4. gewisse Andreiecke eingeführt, deren Inkreise mit dem Umkreis des Kerndreiecks in einer jeweils einfachen Beziehung stehen (Satz 4, Satz 5).

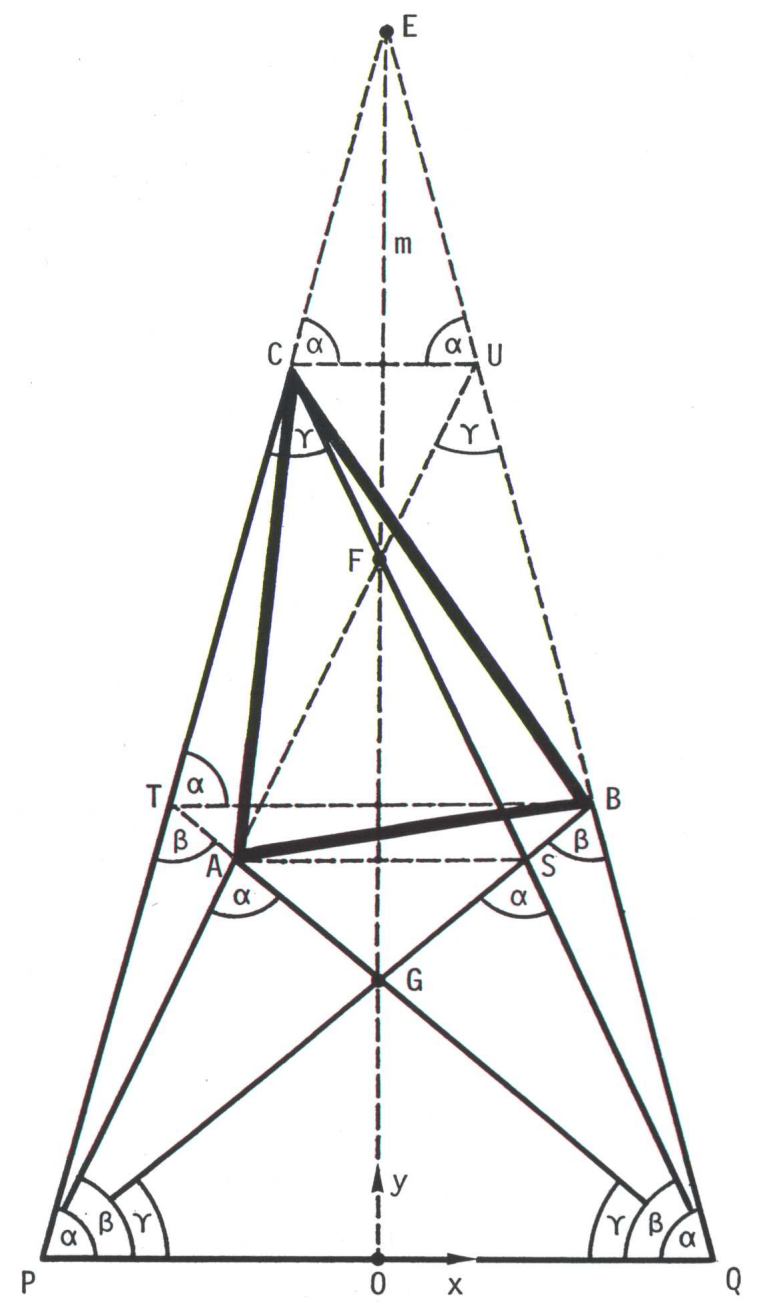

Abbildung 1 


$$
\text { "giering" — 2008/4/9 — 9:45 — page 339 — \#3 }
$$

\section{Beschreibung der Aufsatzfigur}

Einer festen Strecke - der Basis $P Q$ - seien nach derselben Seite die drei gleichsinnig ähnlichen Dreiecke $P Q A, P Q B, P Q C$ aufgesetzt. Die Innenwinkel dieser Aufsatzdreiecke seien (Abb. 1):

$$
\begin{array}{llll}
\frac{P Q A}{\varangle Q A P=\alpha} & \frac{P Q B}{\varangle P Q B=\alpha} & \frac{P Q C}{\varangle C P Q=\alpha} & \\
\varangle A P Q=\beta & \varangle Q B P=\beta & \varangle P Q C=\beta & (\alpha+\beta+\gamma=\pi) . \\
\varangle P Q A=\gamma & \varangle B P Q=\gamma & \varangle Q C P=\gamma &
\end{array}
$$

Im Folgenden betrachten wir den allgemeinen Fall, in dem keine zwei der Winkel $\alpha, \beta, \gamma$ übereinstimmen ${ }^{2}$. In diesem Fall sei ohne Einschränkung $\alpha>\beta>\gamma$. Für die Aufsatzdreiecke $P Q A, P Q B, P Q C$ gilt dann, wenn $m$ das Mittellot der Basis $P Q$ bezeichnet:

In $P Q A$ liegt $A$ links von $m$ (wegen $\beta>\gamma$ ),

in $P Q B$ liegt $B$ rechts von $m$ (wegen $\alpha>\gamma$ ),

in $P Q C$ liegt $C$ links von $m$ (wegen $\alpha>\beta$ ).

Das aus den Ecken $A, B, C$ der Aufsatzdreiecke gebildete Dreieck $A B C$ nennen wir das Kerndreieck der Aufsatzfigur $(P Q, A B C)$. Im Hinblick auf spätere Überlegungen seien in Abbildung 1 die Punkte $E, F, G$ des Mittellots $m$ hervorgehoben. Es sind dies die Schnittpunkte der von $P$ und $Q$ auslaufenden und gegen $P Q$ gleich geneigten (zu Geraden verlängerten) Seiten der Aufsatzdreiecke.

Spiegelt man die Aufsatzdreiecke am Mittelot $m$, so entsteht eine zu $m$ symmetrische Figur. Die an $m$ gespiegelten Ecken $A, B, C$ seien der Reihe nach $S$, $T, U$. Beachtet man die Symmetrie zu $m$ sowie die Parallelitäten $A S\|B T\| C U$ $C U \| P Q$ und die Winkelsumme $\alpha+\beta+\gamma=\pi$, so folgt für die in $A, B, C$ und $S, T, U$ auftretenden Winkel:

$$
\begin{aligned}
& \varangle P S Q=\varangle P A Q=\varangle T B U=\varangle B T C=\varangle C U E=\varangle U C E=\alpha, \\
& \varangle P T Q=\varangle P B Q=\varangle P U C=\varangle Q C U=\varangle A S C=\varangle S A U=\beta, \\
& \varangle P U Q=\varangle P C Q=\varangle P S A=\varangle Q A S=\varangle Q T B=\varangle T B P=\gamma .
\end{aligned}
$$

${ }^{2}$ Wenn zwei der Winkel $\alpha, \beta, \gamma$ übereinstimmen (etwa $\beta=\gamma \neq \alpha$ ), entartet die Aufsatzfigur in leicht durchschaubarer Weise. Dasselbe gilt für $\alpha=\beta=\gamma(=\pi / 3)$. In diesem Sonderfall liegen $A, B, C$ auf dem Mittellot von $P Q$ und es ist $A=B=C$. 


$$
\text { "giering" — 2008/4/9 — 9:45 — page } 340 — \# 4
$$

\section{Ein Streckensatz}

Zur Herleitung eines Streckensatzes beziehen wir die Aufsatzfigur $(P Q, A B C)$ auf das in Abb. 1 eingetragene $x y$-Koordinatensystem. Den Endpunkten der Basis $P Q$ erteilen wir die Koordinaten $P=(-1,0), Q=(1,0)$ und berechnen die Koordinaten der Ecken des Kerndreiecks,

$$
A=\left(x_{1}, x_{2}\right), \quad B=\left(y_{1}, y_{2}\right) \quad C=\left(z_{1}, z_{2}\right),
$$

als die Koordinaten der Geradenschnittpunkte:

$$
\begin{array}{lll}
A=P F \cap Q G, & P F: y=\tan \beta(x+1), & Q G: y=-\tan \gamma(x-1), \\
B=P G \cap Q E, & P G: y=\tan \gamma(x+1), & Q E: y=-\tan \alpha(x-1), \\
C=P E \cap Q F, & P E: y=\tan \alpha(x+1), & Q F: y=-\tan \beta(x-1) .
\end{array}
$$

Unter Verwendung von (5) findet man:

$$
\begin{array}{ll}
x_{1}=\frac{\sin (\gamma-\beta)}{\sin (\gamma+\beta)}, & x_{2}=2 \frac{\sin \gamma \sin \beta}{\sin (\gamma+\beta)}, \\
y_{1}=\frac{\sin (\alpha-\gamma)}{\sin (\alpha+\gamma)}, & y_{2}=2 \frac{\sin \alpha \sin \gamma}{\sin (\alpha+\gamma)}, \\
z_{1}=\frac{\sin (\beta-\alpha)}{\sin (\beta+\alpha)}, & z_{2}=2 \frac{\sin \beta \sin \alpha}{\sin (\beta+\alpha)} .
\end{array}
$$

Wir formulieren nun als erste Eigenschaft der Aufsatzfigur $(P Q, A B C)$ :

SATz 1. Die Längen der von den Endpunkten der Basis $P Q$ auslaufenden Seiten der Aufsatzdreiecke $P Q A, P Q B, P Q C$ genügen den Gleichungen:

$$
P B \cdot Q A=P A \cdot Q C=P C \cdot Q B .
$$

Zum Beweis der Gleichung $P B \cdot Q A=P A \cdot Q C$ entnimmt man aus Abbildung 1:

$$
P B=\frac{y_{2}}{\sin \gamma}, \quad Q A=\frac{x_{2}}{\sin \gamma}, \quad P A=\frac{x_{2}}{\sin \beta}, \quad Q C=\frac{z_{2}}{\sin \beta} .
$$

Ersetzt man in (7) die Koordinaten $x_{2}, y_{2}, z_{2}$ gemäß (6), dann folgt nach elementarer Rechnung $P B \cdot Q A=P A \cdot Q C$. Beachtet man, daß die Aufsatzdreiecke gleichberechtigt sind, dann stellt sich nach zyklischer Vertauschung der Ecken $A$, $B, C$ und Beibehaltung der allen Aufsatzdreiecken gemeinsamen Ecken $P$ und $Q$ die Aussage von Satz 1 ein. 


$$
\text { "giering" — 2008/4/9 — 9:45 — page } 341-\# 5
$$

\section{Kinematische Betrachtungen}

Es sei $k^{\prime}$ ein Kreis durch die bezüglich $m$ symmetrisch liegenden Punkte $A$ und $S$. Der Kreis $k^{\prime}$ schneidet die Gerade $P S$ in $S$ und einem weiteren Punkt $P^{\prime}$ sowie die Gerade $Q S$ in $S$ und einem weiteren Punkt $Q^{\prime}$ (Abb. 2). Dann gilt:

$$
\begin{array}{ll}
\varangle A Q^{\prime} P^{\prime}=\varangle A S P^{\prime}=\gamma \quad\left(k^{\prime} \text { ist Fasskreis über } A P^{\prime} \text { zum Winkel } \gamma\right), \\
\varangle P^{\prime} A Q^{\prime}=\varangle P^{\prime} S Q^{\prime}=\alpha \quad\left(k^{\prime} \text { ist Fasskreis über } P^{\prime} Q^{\prime} \text { zum Winkel } \alpha\right) .
\end{array}
$$

Wegen $\alpha+\beta+\gamma=\pi$ ist $\varangle A P^{\prime} Q^{\prime}=\beta$.

Betrachtet man durch die Punkte $A$ und $S$ anstellte eines festen Kreises $k^{\prime}$ alle Kreise des Kreisbüschels mit den Grundpunkten $A$ und $S$, dann wird eine Schar ähnlicher Dreiecke $P^{\prime} A Q^{\prime}$ definiert mit fester Ecke $A$, mit auf $P B$ laufender Ecke $P^{\prime}$ und auf $Q C$ laufender Ecke $Q^{\prime}$. Die zueinander ähnlichen Dreiecke $P^{\prime} A Q^{\prime}$ liegen drehstreckungsgleich bezüglich $A$ mit dem gegen $A P$ oder $A Q$ gemessenen Drehwinkel $\varphi:=\varangle P A P^{\prime}=\varangle Q A Q^{\prime}$. Die Dreiecke $P A P^{\prime}$ und $Q A Q^{\prime}$ stimmen in den Winkeln $\varphi$ und $\beta-\gamma$ überein und sind ebenfalls drehstreckungsgleich bezüglich $A$. Daher stehen die Dreieckseiten $P P^{\prime}$ und $Q Q^{\prime}$ für alle Drehwinkel $\varphi$ in einem konstanten Verhältnis:

$$
P P^{\prime}: Q Q^{\prime}=P A: Q A=\text { const. }
$$

Wandert bei den Drehstreckungen aus $A$ der auf $P B$ laufende Punkt $P^{\prime}$ nach $B$, so ist in (8) $P^{\prime}=B$. Mit Satz 1 folgt $Q^{\prime}=C^{\prime}$. Damit inzidiert das laufende Dreieck $P^{\prime} A Q^{\prime}$ mit dem Kerndreieck $A B C$ und es gilt:

$$
\varangle P^{\prime} A Q^{\prime}=\varangle B A C=\alpha, \quad \varangle A P^{\prime} Q^{\prime}=\varangle A B C=\beta, \quad \varangle P^{\prime} Q^{\prime} A=\varangle B C A=\gamma .
$$

Da die Aufsatzdreiecke $P Q A, P Q B, P Q C$ nicht gegeneinander ausgezeichnet sind, läßt sich nicht allein das Dreieck $P Q A$ mit den beschriebenen Drehstreckungen um $A$ in das Kerndreieck $A B C$ überführen, sondern auch das Dreieck $P Q B$ mit entsprechenden Drehstreckungen um $B$ und das Dreieck $P Q C$ mit entsprechenden Drehstreckungen um $C$. Neben das Kreisbüschel mit den Grundpunkten $A$ und $S$ treten dann die Kreisbüschel mit den Grundpunkten $B$ und $T$ bzw. $C$ und $U$.

Zusammenfassend erhalten wir:

SATz 2. In der Aufsatzfigur ( $P Q, A B C)$ gilt (Abb. 2):

(a) Das Aufsatzdreieck PQA (PQB bzw. PQC) ist drehstreckungsgleich zum Kerndreieck $A B C$ bezüglich $A$ ( $B$ bzw. $C$ ) als Zentrum der Drehstreckung. 


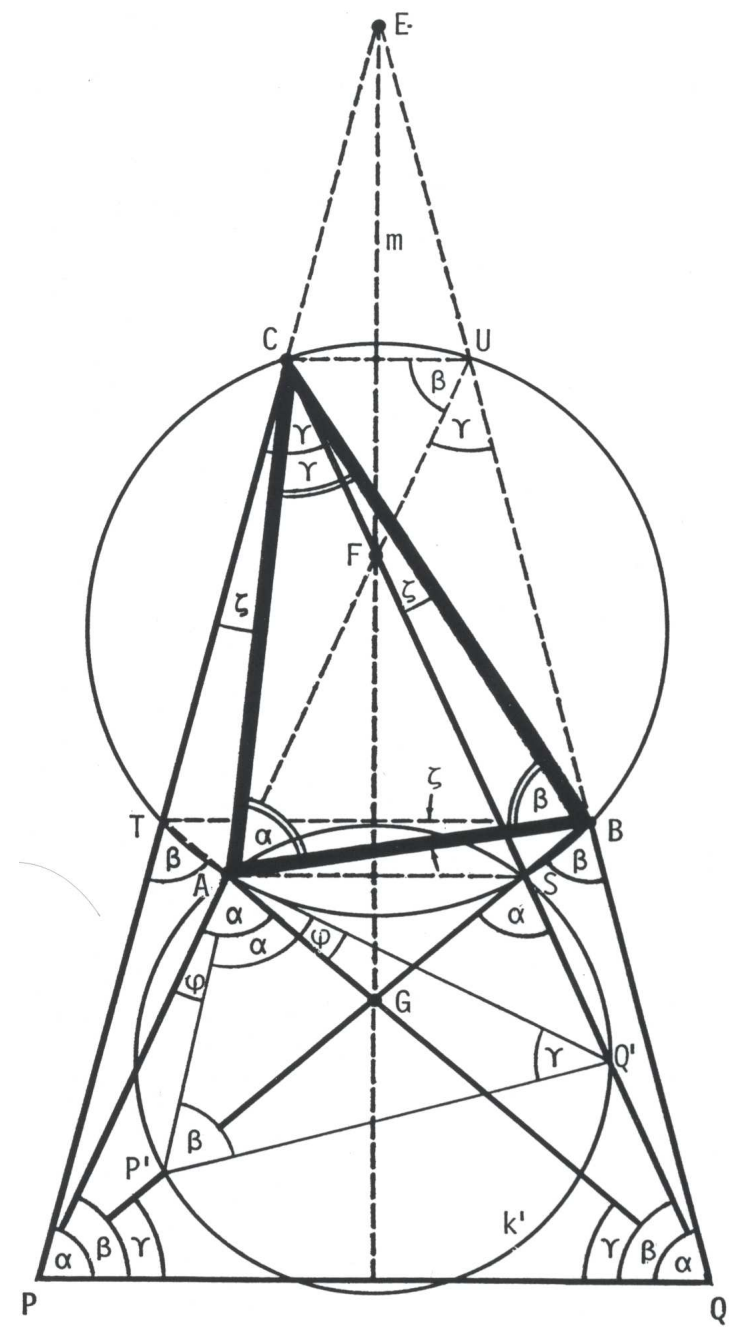

Abbildung 2

(b) Für jeden Kreis $k^{\prime}$ durch die Punkte $A$ und $S$ ist das Dreieck $P^{\prime} A Q^{\prime}$ drehstreckungsgleich zum Aufsatzdreieck $P Q A$ und zum Krendreieck $A B C$. Entsprechendes gilt für jeden Kreis durch die Punkte $B$ und $T$ bzw. $C$ und $U$.

Wir erwähnen in der Aufsatzfigur $(P Q, A B C)$ weitere drehstreckungsgleiche Dreiecke und bemerken zunächst: Die Dreieckseiten $C A$ und $C B$ mit $\varangle A C B=\gamma$ 


$$
\text { "giering" — 2008/4/9 — 9:45 — page } 343 \text { — \#7 }
$$

und die Dreieckseiten $C P$ und $C Q$ mit $\varangle P C Q=\gamma$ sind um $C$ durch den Winkel $\zeta:=\varangle P C A=\varangle Q C B$ gegeneinander gedreht. Man erhält damit:

SATz 3. In der Aufsatzfigur ( $P Q, A B C)$ gilt (Abb. 2):

(a) Die Dreiecke $P A B$ und $Q A C$ stimmen in den Winkeln

$$
\varangle A P B=\varangle A Q C=\beta-\gamma \quad \text { und } \quad \varangle P B A=\varangle Q C A=\gamma-\zeta
$$

überein und sind drehstreckungsgleich bezüglich $A$ als Zentrum der Drehstreckung.

(b) Die Dreiecke $P B C$ und $Q B A$ stimmen in den Winkeln

$$
\varangle B C P=\varangle B A Q=\gamma+\zeta \quad \text { und } \varangle B P C=\varangle B Q A=\alpha-\gamma
$$

überein und sind drehstreckungsgleich bezüglich $B$ als Zentrum der Drehstreckung.

(c) Die Dreiecke PCA und QCB stimmen in den Winkeln

$$
\varangle P C A=\varangle Q C B=\zeta \quad \text { und } \quad \varangle C P A=\varangle C Q B=\alpha-\beta
$$

überein und sind drehstreckungsgleich bezüglich $C$ als Zentrum der Drehstreckung.

\section{Die Andreiecke des Kerndreiecks}

Mit dem Kerndreieck $A B C$ lassen sich die an seine Seiten $A B, B C, C A$ angrenzenden Dreiecke (kurz: Andreiecke) $A B G, B C E, C A F$ verknüpfen (Abb. 3). Bei ihrer Untersuchung dienen die Schnittpunkte des Mittellots $m$ der Basis $P Q$ mit dem Umkreis des Kerndreiecks $A B C$ als Hilfspunkte; sei $V$ der obere und $W$ der untere Schnittpunkt (kurz: $V$ der Hochpunkt, $W$ der Tiefpunkt des Umkreises).

Wir betrachten zunächst das Andreieck $A B G$ mit dem Teildreieck $A B W$ und verwenden die Winkel:

$$
\xi:=\varangle B A G, \quad \eta:=\varangle A B G, \quad \delta:=\varangle B A W, \quad \varepsilon:=\varangle A B W .
$$

Wir beachten, daß gilt:

Der Umkreis des Kerndreiecks $A B C$ ist der Fasskreis für den Winkel $\beta$ über der Sehne $A C$; folglich ist $\varangle A W C=\varangle A B C=\beta$. 


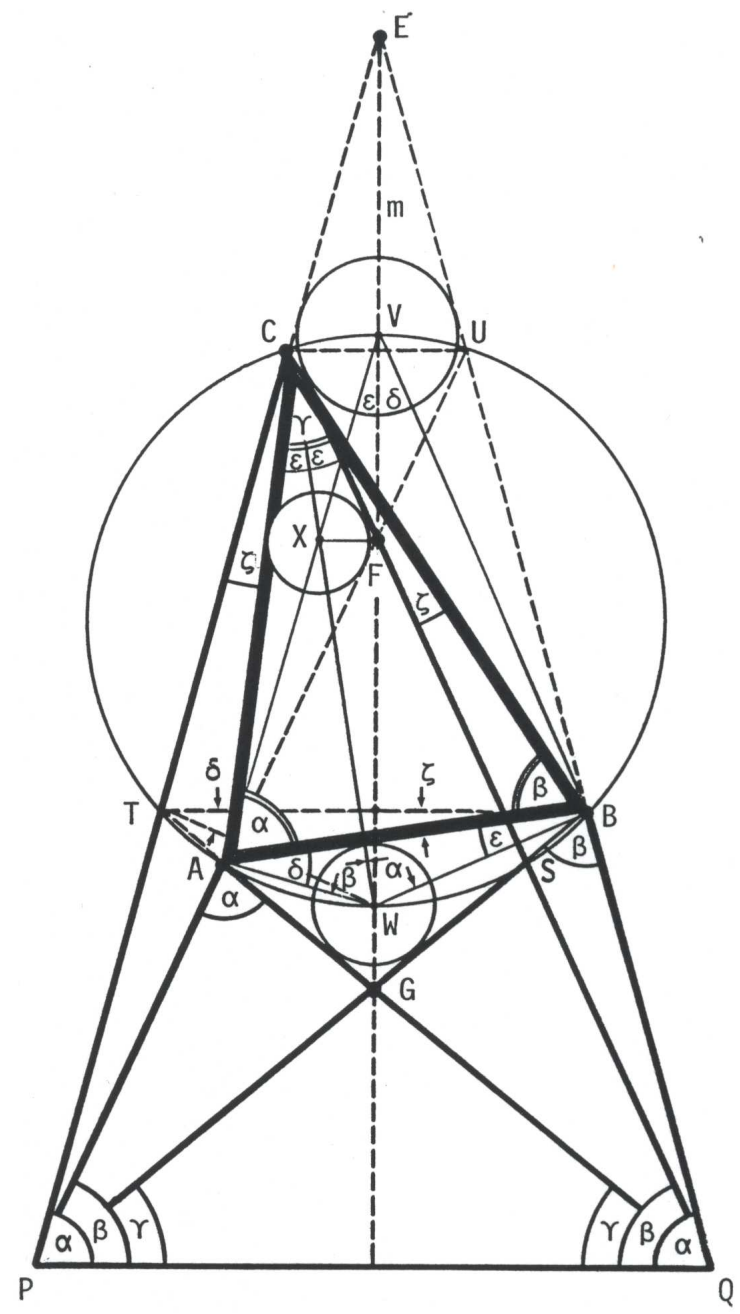

Abbildung 3

Der Umkreis des Kerndreiecks $A B C$ ist auch der Fasskreis für den Winkel $\alpha$ über der Sehne $B C$; folglich ist $\varangle B W C=\varangle B A C=\alpha$. Man erhält dann:

$$
\varangle A W B=\alpha+\beta=\pi-\gamma .
$$

Im Dreieck $A B W$ ist somit die Summe der Innenwinkel

$$
(\pi-\gamma)+\delta+\varepsilon=\pi, \text { also } \delta+\varepsilon=\gamma
$$




$$
\text { "giering" — 2008/4/9 — 9:45 — page 345 — \#9 }
$$

Im gleichschenkligen Dreieck $P G Q$ findet man $\varangle P G Q=\varangle A G B=\pi-2 \gamma$. Im Andreieck $A B G$ ist somit die Summe der Innenwinkel

$$
(\pi-2 \gamma)+\xi+\eta=\pi, \text { also } \xi+\eta=2 \gamma
$$

Aus (12) und (13) folgt

$$
(\xi-2 \delta)+(\eta-2 \varepsilon)=0 .
$$

Wir beachten nun: Der Umkreis des Kerndreiecks $A B C$ ist der Fasskreis für den Winkel $\delta$ über der Sehne $W B$. Daher ist $\varangle W T B=\delta$. Wegen der Symmetrie des Dreiecks $T W B$ zum Mittellot $m$ der Basis $P Q$ ist $\varangle W T B=\varangle W B T=\delta$.

Wir beachten weiter: Der Umkreis des Kerndreiecks $A B C$ ist der Fasskreis für den Winkel $\zeta:=\varangle T C A=\varangle T B A=\varangle B A S$.

Nun gilt im Dreieck $P A C$ einerseits

$$
\varangle P A C=\pi-\zeta-(\alpha-\beta)
$$

und anderseits

$$
\varangle P A C=2 \pi-2 \alpha-\xi
$$

Aus (15) und (16) folgt

$$
\xi=\gamma+\zeta
$$

Ersetzt man in (17) den Winkel $\zeta$ durch $\zeta=\varangle T B A=\delta-\varepsilon$, so folgt

$$
\xi=2 \delta \text {. }
$$

Nach (18) ist im Andreieck $A B G$ die Gerade $A W$ die Innenwinkelhalbierende in $A$. Da die Dreieckseiten $A G$ und $B G$ zum Mittellot $m=G W$ der Basis $P Q$ symmetrisch liegen, ist $G W$ im Andreieck $A B G$ die Innenwinkelhalbierende in $G$. Als Schnittpunkt zweiter Innenwinkelhalbierenden ist $W$ der Inkreismittelpunkt des Andreiecks $A B G$.

Im Andreieck $B C E$ halbieren die Geraden $B V, C V$ und $E V$ die Innenwinkel. Der Hochpunkt $V$ des Umkreises des Kerndreiecks $A B C$ ist folglich der Inkreismittelpunkt des Andreiecks BCE.

Wir erhalten:

SATz 4. In der Aufsatzfigur ( $P Q, A B C)$ gilt (Abb. 3):

(a) Der Tiefpunkt $W$ des Umkreises des Kerndreiecks $A B C$ ist der Inkreismittelpunkt des Andreiecks $A B G$.

(b) Der Hochpunkt $V$ des Umkreises des Kerndreiecks $A B C$ ist der Inkreismittelpunkt des Andreiecks BCE. 


$$
\text { "giering" — 2008/4/9 — 9:45 — page 346 — \#10 }
$$

In dem noch ausstehenden Andreieck $C A F$ gilt:

Die Geraden $A F$ und $C F$ sind neigungsgleich gegen die Basis $P Q$; die $P Q$ Parallele durch $F$ halbiert daher den Winkel $\varangle C F A$.

Die Gerade $C W$ halbiert den Winkel $\varangle A C F$. Zum Beweis notieren wir: Im Dreieck $A C W$ gilt:

$$
\varangle A C W+(\alpha+\delta)+\beta=\pi \text {. Wegen } \alpha+\beta+\gamma=\pi \text { folgt } \varangle A C W=\gamma-\delta \text {. }
$$

Im Dreieck $B C W$ gilt:

$(\varangle W C F+\zeta)+(\beta+\varepsilon)+\alpha=\pi$. Wegen $\alpha+\beta+\gamma=\pi$ folgt $\varangle W C F=\gamma-(\zeta+\varepsilon)$.

Addiert man in (12) auf beiden Seiten der Gleichung $\gamma=\delta+\varepsilon$ den Winkel $\delta$, so folgt:

$$
\delta=2 \delta-\gamma+\varepsilon \stackrel{(18)}{=} \xi-\gamma+\varepsilon \stackrel{(17)}{=} \zeta+\varepsilon .
$$

Mit (21) liefern (19) und (20): $\varangle A C W=\varangle W C F$. Die Gerade $C W$ halbiert also den Winkel $\varangle A C F$ und schneidet folglich die $P Q$-Parallele durch $F$ im Inkreismittelpunkt $X$ des Andreiecks $C A F$.

Wir beachten schließlich in der Aufsatzfigur $(P Q, A B C)$ die Winkelgleichheiten:

$$
\varangle C A V=\varangle C W V=\varangle U W V=\varangle U A V=\varangle F A V .
$$

Zum Beweis interpretiere man den Umkreis des Kerndreiecks $A B C$ als Fasskreis und verwende, daß die Winkel $\varangle C W V$ und $\varangle U W V$ zum Mittellot $m$ der Basis $P Q$ symmetrisch liegen. Nach (22) halbiert die Gerade $A V$ den Winkel $\varangle C A F$ und läuft daher durch den Inkreismittelpunkt $X$ des Andreiecks CAF.

Wir erhalten:

Satz 5. In der Aufsatzfigur ( $P Q, A B C)$ gilt (Abb. 3): Der Inkreismittelpunkt $X$ des Andreiecks $C A F$ ist der Schnittpunkt der Geraden $A V$ und $C W ; V$ ist der Hochpunkt, $W$ der Tiefpunkt des Umkreises des Kerndreiecks $A B C$.

Bemerkung. Das Mittellot $m$ der Basis $P Q$ halbiert im Andreieck $A B G$ den Winkel $\varangle A G B$, im Andreieck $B C E$ den Winkel $\varangle B E C$, jedoch nicht im Andreieck $C A F$ den entsprechenden Winkel $\varangle C F A$. Insofern sind die Andreiecke $A B G$, $B C E, C A F$ nicht gleichberechtigt. Ein Licht auf diese Situation wirft das Axiom von Pasch. Es besagt anschaulich - angewandt auf das Mittellot $m$ - nach [5], S. 5: Wenn $m$ (in einem Punkt zwischen $A$ und $B$ als Halbierende des Winkels $\varangle A G B$ ) ins Innere des Kerndreiecks $A B C$ eintritt, dann tritt $m$ auch wieder heraus (in 


$$
\text { "giering" — 2008/4/9 — 9:45 — page } 347 \text { — \#11 }
$$

der Aufsatzfigur in einem Punkt zwischen $B$ und $C$ als Halbierende des Winkels $\varangle B E C)$. Das Mittellot $m$ kann dann das Innere des Kerndreiecks $A B C$ nicht auch noch in einem Punkt zwischen $C$ und $A$ verlassen, kann also den Winkel $\varangle C F A$ nicht halbieren. Dies besorgt die zu $m$ orthogonale Gerade durch $F$.

\section{Ein Kopunktalitätssatz}

Wir betrachten abschließend die drei Schwerlinien der Aufsatzdreiecke $P Q A$, $P Q B, P Q C$, die jeweiles den Scheitel ihres Innenwinkels $\sigma(\sigma \in\{\alpha, \beta, \gamma\})$ enthalten; wir nennen diese Schwerlinien die $\sigma$-Schwerlinien der Aufsatzdreiecke. Ermittelt man aus den in Abschnitt 2 angegebenen Koordinaten der Punkte $O$, $P, Q$ und $A, B, C$ (siehe (6)) die Gleichungen der $\sigma$-Schwerlinien und testet man ihre Kopunktalität, so folgt

Satz 6. In der Aufsatzfigur $(P Q, A B C)$ sind die drei $\sigma$-Schwerlinien der Aufsatzdreiecke $P Q A, P Q B, P Q C$ für $\sigma \in\{\alpha, \beta, \gamma\}$ jeweils kopunktal.

Bemerkung. Betrachtet man die in $O$ kopunktalen Schwerlinien $O A, O B$, $O C$ der Aufsatzdreiecke sowie deren Schwerpunkte $S_{A} \in O A, S_{B} \in O B, S_{C} \in$ $O C$, dann besteht nach einer elementaren Schwerpunkteigenschaft das Streckenverhältnis

$$
O S_{A}: O A=O S_{B}: O B=O S_{C}: O C=1: 3 .
$$

Folglich bilden die Schwerpunkte $S_{A}, S_{B}, S_{C}$ der Aufsatzdreiecke $P Q A, P Q B$, $P Q C$ ein zum Kerndreieck $A B C$ zentrisch ähnliches Dreieck mit dem Ähnlichkeitszentrum $O$.

\section{Literatur}

[1] G. Berkhan, W. Fr. Meyer, Neuere Dreiecksgeometrie, in: Encyklopädie der mathematischen Wissenschaften, Bd. 3, AB9, Teubner Leipzig (1914-1931), 1173-1276.

[2] H. S. M. Coxeter, S. L. Greitzer, Zeitlose Geometrie, Klett Stuttgart, 1983.

[3] J. Fischer, Napoleon und die Naturwissenschaften, Steiner Stuttgart, 1988.

[4] O. Giering, Zur Theorie der Aufsatzdreiecke, IBDG 26 (2007), 34-41.

[5] D. Hilbert, Grundlagen der Geometrie, 8. Aufl., Teubner Stuttgart, 1956.

[6] H. Martini, On the theorem of Napoleon and related topics, Math. Semesterberichte 43 (1996), 47-64. 
[7] H. Schaal, Ein Beitrag zur Geometrie ähnlich veränderlicher Felder, El. Math. 21 (1966), 97-109.

OSWALD GIERING

TU MÜNCHEN, ZENTRUM MATHEMATIK

D-85748 GARCHING

UND

JOHANN-STRAUSS-STR. 30

D-85591 VATERSTETTEN

DEUTSCHLAND

(Received May, 2007) 